\title{
Planetary Nebulae and Wolf-Rayet Nebulae
}

\author{
Y. Grosdidier ${ }^{a, b}$ \\ ${ }^{a}$ Instituto de Astrofísica de Canarias, Calle Vía Láctea s/n, E-38200 La \\ Laguna (Tenerife), Spain \\ ${ }^{b}$ Observatoire Astronomique de Strasbourg, UMR 7550, 11 rue de \\ l'Université, F-67000 Strasbourg, France
}

\begin{abstract}
In the present paper I review some important facts regarding the similarities and differences between Galactic planetary nebulae and Galactic Wolf-Rayet (WR) nebulae within the scope of stellar mass-loss history and its subsequent impact on the nebular dynamics and morphology. The case of planetary nebulae with [WR] nuclei, which allows one to perform a more direct comparison with WR nebulae, is emphasized. In particular, I describe the apparently ubiquitous turbulent-like phenomena originating in [WR] stellar atmospheres and the surrounding nebulae, and discuss the possible impact of turbulence on planetary nebula studies.
\end{abstract}

\section{Introduction}

Stars interact with the surrounding interstellar medium (ISM) both through their ionizing radiation and through the mass, momentum and energy that are transferred by way of their winds. The ultraviolet radiation originating in hot stars leads to ionized nebulae ('HII regions' in the broad sense) around massive stars, or planetary nebulae (PNe) in the case of low-mass stars.

Nebulae around massive Wolf-Rayet (WR) stars (hereafter 'WR nebulae'), which represent a late phase in the evolution of massive stars with initial masses above $35-40 \mathrm{M}_{\odot}{ }^{5}$, and around PNe share many physical properties and formation mechanisms. In this paper I discuss the similarities between these two types of nebulae with an emphasis on PNe surrounding [WR] central stars, which allows one to perform a more direct comparison with WR nebulae. As is well known, it turns out that these similarities are tightly related to the mass-loss histories experienced by both types of hot stars. For detailed considerations on the formation and evolution of $\mathrm{PNe}$, the reader is referred to other papers, in particular those presented in these proceedings by Schönberner \& Steffen, Mellema, Tylenda, Hamann, and García-Segura.

\footnotetext{
${ }^{5}$ Note that the minimum mass required to form a non-binary population I WR star may be significantly smaller $\left(\approx 25 \mathrm{M}_{\odot}\right)$ when stellar rotation effects are taken into account (Maeder 2000 and references therein).
} 


\section{Planetary nebulae and Wolf-Rayet nebulae: mass-loss history}

As was already pointed out by Chu (1993), nebulae around central stars of PNe and massive WR stars prove to be a powerful diagnostic tool to understand how mass is lost in the post main-sequence phases of stellar evolution. Central stars of $\mathrm{PNe}$ and a relatively large fraction $(\approx 35 \%$; Marston 1999 and references therein) of massive WR stars are surrounded by circumstellar nebulae made up of stellar material ejected during successive stellar wind phases (see Table 1). These nebulae often appear to have similar physical properties, which point to a common formation mechanism, independent of the strong differences between both types of hot stars. The next two sections are intended to remind the reader of i) the similarities of population I \& II WR radiatively driven stellar winds and, ii) the similarities expected in the context of the interacting stellar wind model (ISWM), since similar successive stellar wind phases lead to comparable wind-wind collisions for both populations.

\begin{tabular}{|c|l|}
\hline Planetary Nebula Central Star Mass-Loss History \\
\hline AGB star & $v_{\infty} \sim 10^{1} \mathrm{~km} \mathrm{~s}^{-1}$ \\
$\downarrow$ & $\dot{M} \sim 10^{-7}-10^{-4} \mathrm{M}_{\odot} \mathrm{yr}^{-1}$ \\
\cline { 2 - 2 } Central Star of PN & $v_{\infty} \sim 10^{3} \mathrm{~km} \mathrm{~s}^{-1}$ \\
& $\dot{M} \sim 10^{-9}-10^{-7} \mathrm{M}_{\odot} \mathrm{yr}^{-1}$ \\
\hline \multicolumn{2}{|c|}{ Massive WR Star Mass-Loss History } \\
\hline Main Sequence O-star & $v_{\infty} \sim 10^{3} \mathrm{~km} \mathrm{~s}^{-1}$ \\
$\downarrow$ & $\dot{M} \sim 10^{-11}-10^{-5} \mathrm{M}_{\odot} \mathrm{yr}^{-1}$ \\
\cline { 2 - 2 } RSG or LBV stage & $v_{\infty} \sim 10^{1}-10^{2} \mathrm{~km} \mathrm{~s}^{-1}$ \\
$\downarrow$ & $\dot{M} \sim 10^{-5}-10^{-4} \mathrm{M}_{\odot} \mathrm{yr}^{-1}$ \\
\cline { 2 - 2 } & $v_{\infty} \sim 10^{3} \mathrm{~km} \mathrm{~s}^{-1}$ \\
WR star & $\dot{M} \sim 10^{-6}-10^{-4} \mathrm{M}_{\odot} \mathrm{yr}^{-1}$ \\
\hline \hline
\end{tabular}

Table 1. Qualitative, main mass-loss intervals experienced by massive WR stars and central stars of PNe during their evolution.

\subsection{Wolf-Rayet stellar winds}

For both population I \& II WR stars, line formation is normally assumed to arise in a spherically expanding atmosphere, where non-LTE conditions (e.g. Hillier 1990; Koesterke 2001, and references therein) prevail. Moreover, population I \& II WR stars of the same subtype are virtually indistinguishable on the basis of their stellar spectra alone (see bottom panels of Figure 1, and Méndez et al. 1991). This can be heuristically understood through consideration of the Sobolev optical depth $\left(\tau_{S o b} \sim \rho(d v / d r)^{-1}\right)$ in both contexts. Assuming a stationary, spherical mass-loss $\left(\dot{M}=4 \pi r^{2} \rho v\right)$ and a velocity field close to that of the so-called $\beta$-law $\left(v(r)=v_{\infty}\left(1-R_{*} / r\right)^{\beta}\right)$ with $\beta \sim 1$, the optical depth of the P-Cygni profiles roughly scales as $\tau_{S o b} \sim \dot{M} v_{\infty}^{-2} R_{*}^{-1}$ (provided that the distance from the stellar surface is large enough). Therefore (see Table 1), taking into account the fact that population I \& II WR stellar winds have similar terminal velocities and that central star radii are about 10-100 times smaller than those of massive WR stars, typical central stars of PNe with $\dot{M}=10^{-8} \mathrm{M}_{\odot} \mathrm{yr}^{-1}$ 

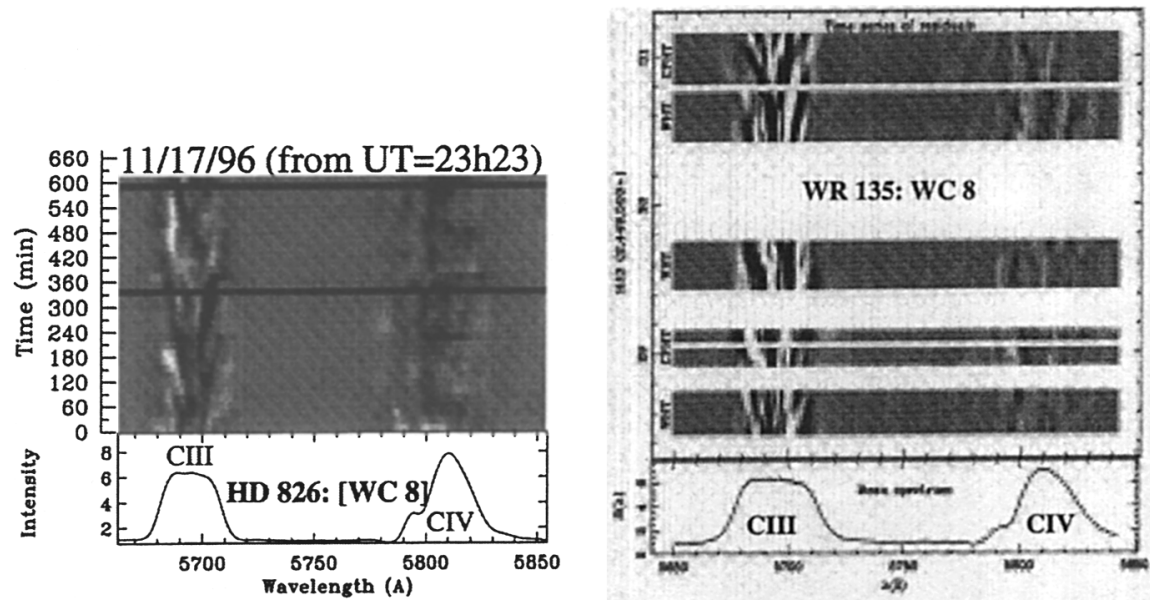

Figure 1. Spectroscopic time-series of NGC 40's central star $(=\mathrm{HD}$ 826, [WC 8]; left panel) and WR 135 (=HD 192103, WC 8; right panel). The mean profiles for the whole data sets are also shown (bottom panels). The figures are adapted from Grosdidier et al. (2001a) and Lépine et al. (2000).

have about the same Sobolev optical depths as a massive WR with $\dot{M}=10^{-6}$ $\mathrm{M}_{\odot} \mathrm{yr}^{-1}$.

Consequently, one needs additional criteria in order to discriminate between population I \& II WR stars: e.g. absolute luminosities or linear radii of the surrounding nebulae (both are significantly smaller for population II WR stars). An exemplary case illustrating the real confusion between population I \& II WR stars is that of the WR nebula M 1-67, surrounding the WN 8 star WR 124 (Chu 1993; Grosdidier et al. 2001b). On the other hand, recall that no clear and non-ambiguous WN central star of a PN has been detected so far in our Galaxy. Therefore, a Galactic WR star belonging to the nitrogen sequence is very likely a massive WR rather than a central star of a PN. Although the similarities in line profiles suggest that the winds of [WR] central stars are scale models of massive WR stellar winds, the level of excitation conditions among WR central stars is quite different since it spans a large range, from [WC 4] to [WC 11] and from [WO 1] to [WO 4] (Méndez \& Niemela 1982; Hu \& Bibo 1990; van der Hucht 1996, Acker \& Durand 2002), compared to WC 4-WC 9 (with extension to WO 1-WO 4 at the hot-end) for massive, population I WR stars (van der Hucht 2001).

The cool subclasses of population II [WR] stars are close to the entry point into the WR phase, subsequent evolution likely occurring to earlier, hotter subtypes via the mass-loss peeling-off process and/or convective mixing and subsequent burning: [WC]-late $\rightarrow$ [WC]-early ${ }^{6}$ (Koesterke 2001; Acker et al. 1996). Massive, population I WR stars of the carbon sequence are believed to follow

${ }^{6}$ However, this scheme is still somewhat controversial (Peña et al. 2001, and references therein). 

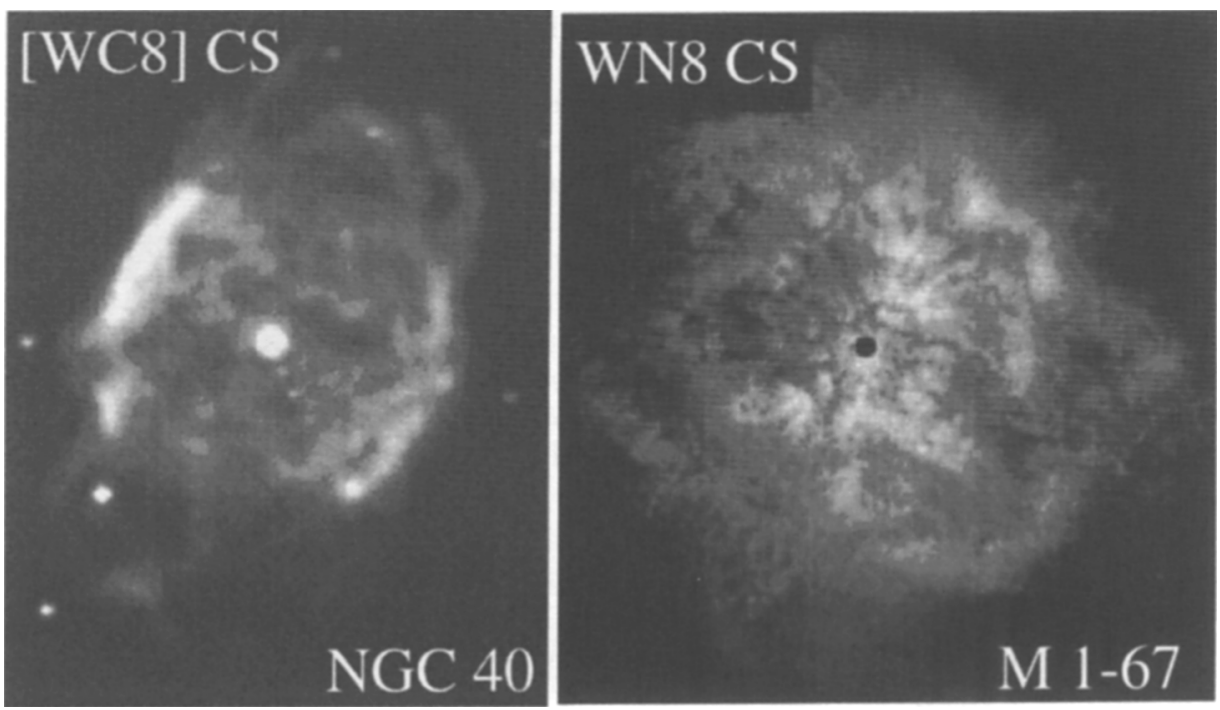

Figure 2. $\mathrm{H} \alpha$ images of a sample Galactic PN (NGC 40; Credit: NOAO/AURA/NSF, (CWIYN Cons., Inc., all rights res.), and a Galactic WR nebula (M 1-67/WFPC2; Grosdidier et al. 2001b).

a similar evolutionary scenario, WC-late $\rightarrow$ WC-early (Moffat 1995), although the Galactocentric distribution per subtype does not encourage this scheme (van der Hucht 2001).

\subsection{Wind-wind collisions and the interacting stellar wind model}

In the context of the ISWM (originally proposed to explain PN formation and dynamics; Kwok et al. 1978), a spherically symmetric, fast, hot stellar wind is catching up and colliding with a pre-existing slower, denser wind. Despite the presence of an extra (generally very large) wind-blown cavity, believed to have been produced by the original main sequence O-star progenitor in the case of massive WR stars, Table 1 suggests that the ISWM can be successfully applied to explain PN and WR nebula formations. This is indeed the case as demonstrated by several studies (PN: Kwok et al. 1978; Balick 1994; WR nebulae: Marston 1999; García-Segura, Mac Low \& Langer 1996, and references therein). Figure 2 demonstrates obvious morphological similarities between PN and WR nebulae (M 1-67 being about 10 times physically larger than NGC 40).

The non-circular shapes of many PN or WR nebulae suggest that the fast wind is sweeping up a slow wind that is flattened by rotation or binary effects. Rotation acts by forming equatorial density enhancements in winds from stars rotating near the critical velocity for liftoff of material from the equator. Subsequent faster winds expanding into the resulting axisymmetric density distributions will form non-spherical nebulae. On the other hand, a flattened, fast wind blowing out a spherically symmetric slow wind can also lead to a non-circular nebula. Magnetic fields act when toroidal fields in the wind contract to colli- 
mate the flow toward the axis, producing the ansae and jets observed in some $\mathrm{PNe}$ (in the case of massive WR, no clear evidence for magnetic fields has been reported so far). On the whole, the combined effects of rotation and magnetic fields, along with photoionization, can explain the rich variety of observed PN shapes in a natural and self-consistent way (García-Segura 2002).

Note that the evolutionary scenarios presented in Table 1 are much too simple. Indeed, one often needs to consider additional transitional mass-loss phases in order to understand how asymmetries may appear and develop. For instance, while AGB stars display spherical shapes and winds, PNe appear asymmetric. By studying proto-planetary nebulae (PPNe), we can learn about when and how these asymmetric shapes develop. Young PNe and PPNe appear highly aspherical, with complex multipolar morphologies. The central star is often offset from the geometrical symmetry-centres of various nebular components. On the other hand, bright aspherical nebulae are often found to be surrounded by faint, roughly round halos, which are the signatures of the progenitor AGB envelopes produced by isotropic mass-loss. A number of these halos include numerous concentric arcs, evidence for quasi-periodic modulation of the mass-loss on time-scales of a few hundred years. On the whole, detailed imaging studies of PPNe show the emergence of complex morphological structures during the PPN phase (Hrivnak, these proceedings).

\section{Wolf-Rayet central stars and massive Wolf-Rayet stars: stellar wind structures}

For both population I \& II WR stars, line formation is assumed to arise in a spherically expanding atmosphere, where similar intricate radiative/hydrodynamic instabilities prevail (Dessart \& Owocki 2002, and references therein). Recall that the spectral variability originating in massive WR stars (Schumann \& Seggewiss 1975; Lépine \& Moffat 1999, and references therein) and central stars of PNe shows the WR phenomenon to be remarkably similar in both (Grosdidier et al. 2000, 2001a; Acker \& Grosdidier 2002, and references therein). Despite the strong differences between both types of hot stars, this supports the understanding of the WR spectral phenomenon as being purely atmospheric. The moving subpeaks that are systematically seen on the tops of broad optical emission lines from population I \& II WR stars suggest that WR winds are inhomogeneous and non-stationary (on a time-scale of hours). See Figure 1, upper panels.

Furthermore, evidence is mounting that all fast, hot winds are potentially inhomogeneous. Time series of very high signal-to-noise ratio, high-resolution spectra of the HeII $\lambda 4686$ emission line in the O4I(n)f supergiant $\zeta$ Puppis have been obtained by Eversberg et al. (1998). In this study, the authors detected stochastic, variable substructures in the line, like those observed in the winds of massive WR stars, which are probably the descendents of $\mathrm{O}$ and Of stars. Therefore, evidence is accumulating that wind clumping may be an intrinsic characteristic of all radiatively driven stellar winds. Moreover, one may expect hydrogen-deficient [WR] winds to be extreme examples for central stars of PNe, so that any fine structure found in [WR] winds may apply to all winds of central stars of $\mathrm{PNe}$, much as one is finding now that weak, massive O-star winds also show the same fine structure as massive WR winds. 


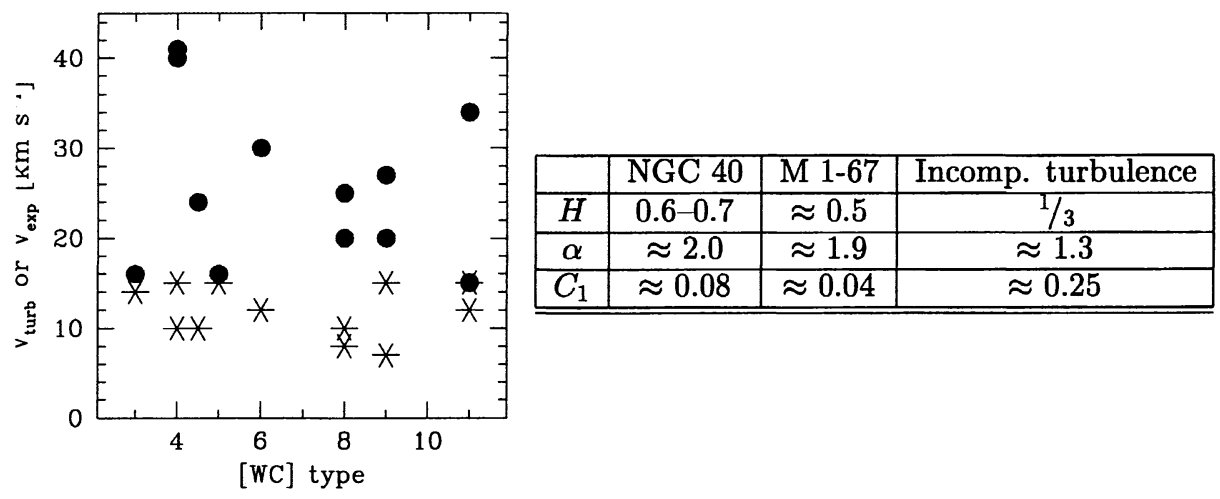

Figure 3. Left panel: Expansion (dots) and turbulent (asterisks) velocities plotted against [WC] spectral type (Acker et al. 2002). Right panel: multifractal indices found for the NGC 40 and M 1-67 nebulae (Grosdidier et al. 2001b, 2002). The related values for incompressible turbulence are the main results of Schmitt et al. (1992).

\section{Turbulent motions in ejected nebulae}

Population I \& II WR stellar winds exhibit strong interaction with their environment, for example with wind outflow from a previous stellar evolutionary stage, and/or with the surrounding ISM. In addition, in both types of interactions, the interface is subject to various kinds of instabilities. Moreover, the material in these outflows interacts on a variety of spatial and temporal scales, leading to lively, patchy complex nebulae (see Figure 2) whose structure and dynamics are far from being reminiscent of simple expanding thick shells.

On the whole, these hydrodynamical instabilities are expected to lead to turbulence in the nebulae. On a qualitative basis, the interacting stellar wind model agrees with the observations, but it is still difficult to determine the precise initial, overall geometry of the colliding winds. In particular, the question arises as to the impact of pre-collision, apparently universal, clumping or possible turbulence of the hot-star winds (Lépine \& Moffat 1999; Grosdidier et al. 2000, 2001a, and references therein) on the dynamics/morphology of circumstellar nebulae.

Taking into account the fact that [WR] stellar winds are more massive, highly H-deficient and metal enriched compared to the winds of normal central stars of $\mathrm{PNe}$, it is useful to recall Mellema (2002) who pointed out that $\mathrm{PNe}$ around [WR] central stars should reveal faster expansion velocities and a longer momentum-driven phase, potentially leading to more turbulent nebulae. The latter point was strongly supported observationally by Acker et al. (2002) who found systematic turbulent velocities originating in PNe around [WR] stars (Figure 3, left panel), while the nebulae around O-type stars show no clear evidence for turbulent motions. Although stellar wind clumps cannot reach large nebular distances easily (Runacres \& Owocki 2002), they can play an important role during the momentum-driven phase, which is known to be very sensitive to 
the non-linear thin-shell instability, as well as to other hydrodynamical instabilities. Therefore, the detected turbulence could be either triggered, or enhanced, by stellar wind clumps that appear ubiquitous in population I \& II WR stellar winds.

Note that the turbulent status in PN and WR nebulae could be investigated through the study of the statistical properties of fluctuating gas motions using structure functions (Frisch 1995) traced by nebular emission-line centroid velocities. Additionally, we can discuss the nature of the turbulence in terms of Universal Multifractals, a continuous-scale limit of multiplicative cascades (Schertzer \& Lovejoy 1987) and derive the level of intermittency in the nebulae. Indeed, it turns out that turbulent, multiplicative cascade processes can be generated from a limited number of universal parameters: $0<\alpha \leq 2$, the basic parameter which characterizes the process $(\alpha=2$ corresponds to the log-normal distribution, a gaussian 'generator'), $C_{1}$, which quantifies the sparseness of the mean field $\left(C_{1}\right.$ close to 0 implies nearly homogeneous fields), and $H$, which quantifies the degree of non-conservativity of the field (Grosdidier et al. 2001b, 2002 , and references therein).

Such an approach has been done so far for only two objects, NGC 40, and M 1-67, and additionally allows one to compare the results with turbulent velocity measurements in wind tunnel experiments (leading to Kolmogorov, incompressible turbulence); see Figure 3, right panel. Because the $H$ parameter is known to be affected by smearing effects along the line of sight (O'Dell \& Castañeda 1987), we expect to measure different $H$ values for various objects. On the other hand, in the case of turbulence driven by stellar winds, the multifractal parameters $\alpha$ and $C_{1}$ are likely to take on universal values. The results shown in the right panel of Figure 3 support this hypothesis. The higher degree of multifractality and the smaller intermittency found for NGC 40 and M 1-67 compared to Kolmogorov turbulence is likely a consequence of the compressible nature of the tracing gas.

\section{Some concluding remarks}

Beyond the obvious morphological and dynamical similarities between Galactic planetary nebulae and Wolf-Rayet nebulae, we have pointed out that both the central stars and the surrounding nebulae are affected by apparently ubiquitous, turbulent-like motions. We suspect that, if more data are secured for larger samples of objects, turbulent phenomena in population I \& II stellar winds and their related circumstellar nebulae may reveal themselves to be more common and universal. The consequences of clumping in population I \& II WR winds are manifold, including substantial constraints on the effective mass-loss rates, and their possible impact on the surrounding nebulae themselves. In addition, stellar wind clumping allows one to study astrophysical turbulence for its own sake on tractable time-scales. On the other hand, turbulence studies in the related nebulae provide a potential way to estimate temperature fluctuations in PNe and WR nebulae and any HII regions (see Nordlund \& Padoan 1999 for a discussion on the log-normality of the density field in isothermal plasmas). The latter approach might be an effective way to account for turbulence in the chemical models of PN and WR nebulae in a realistic way. 


\section{References}

Acker, A., \& Durand, S. 2002, these proceedings

Acker, A., Gesicki, K., Grosdidier, Y., Durand, S. 2002, A\&A, 384, 620

Acker, A., Gorny, S.K., Cuisinier, F. 1996, A\&A, 305, 949

Acker, A., \& Grosdidier, Y. 2002, these proceedings

Balick, B. 1994, Ap\&SS, 216, 13

Chu, Y.-H. 1993, IAU Symp. 155, p. 139

Dessart, L., \& Owocki, S.P. 2002, A\&A, 383, 1113

Eversberg, T., Lépine, S., Moffat, A.F.J. 1998, ApJ, 494,799

Frisch, U. 1995, Turbulence, Cambridge Univ. Press

García-Segura, G. 2002, these proceedings

García-Segura, G., Mac Low, M.-M., Langer, N. 1996, A\&A, 305, 229

Grosdidier, Y., Acker, A., Blais-Ouellette, S., et al. 2002, these proceedings

Grosdidier, Y., Acker, A., Moffat, A.F.J. 2000, A\&A, 364, 597

Grosdidier, Y., Acker, A., Moffat, A.F.J. 2001a, A\&A, 370, 513

Grosdidier, Y., Moffat, A.F.J., Blais-Ouellette, S., et al. 2001b, ApJ, 562, 753

Hillier, J. 1990, A\&A, 231, 111

Hu, J.Y., \& Bibo, E.A. 1990, A\&A, 234, 435

van der Hucht, K.A. 1996, Ap\&SS, 238, 1

van der Hucht, K.A. 2001, New Astron.Rev., 45, 135

Koesterke, L. 2001, Ap\&SS, 275, 41

Kwok, S., Purton, C.R., FitzGerald, P.M. 1978, ApJ, 219, L125

Lépine, S., \& Moffat A.F.J. 1999, ApJ, 514, 909

Lépine, S., et al. 2000, AJ, 120, 3201

Maeder, A. 2000, ARA\&A, 38, 143

Marston, A.P. 1999, IAU Symp. 193, p. 306

Mellema, G. 2002, these proceedings

Méndez, R.H., Herrero, A., Manchado, A., Kudritzki, R.P. 1991, A\&A, 252, 265

Méndez, R.H., \& Niemela, V.S. 1982, IAU Symp. 99, p. 457

Moffat, A.F.J. 1995, IAU Symp. 163, p. 213

Nordlund, A., \& Padoan, P. 1999, Interstellar Turbulence, Proc. of the $2^{\text {nd }}$ G. Haro Conf., Eds. J. Franco \& A. Carraminana. Cambridge Univ. Press, p. 218

O’Dell, C.R., \& Castañeda, H.O. 1987, ApJ, 317, 686

Peña, M., Stasińska, G., Medina, S. 2001, A\&A, 367, 983

Runacres, M.C., \& Owocki, S.P. 2002, A\&A, 381, 1015

Schertzer, D., \& Lovejoy, S. 1987, J. Geophys. Res., 92, 9693

Schmitt, F., Lavallée, D., Schertzer, D., et al. 1992, Phys.Rev.Lett, 68, 305

Schumann, J.D., \& Seggewiss, W. 1975, IAU Symp. 67, p. 299 